\title{
Integrated product model centred design in a virtual design office
}

\author{
R.J. Scherer and P. Katranuschkov \\ Prof. Dr.-Ing.; Senior research assistant \\ Institute of structural mechanics and applied computer science, \\ Dresden Univ. of Technology, D- 01062 Dresden, Germany, \\ E-mail: scherer@cib.bau.tu-dresden.de
}

\begin{abstract}
The prototype software environment developed in the EU ESPRIT project 6609 COMBI demonstrates in practice the benefits of open system integration for the structural engineering domain. The environment is based on an object-oriented product modelling framework conforming to the ISO STEP methodology. This paper discusses the main concepts realised in the COMBI project w.r.t. product modelling and interoperability in order to enable co-operative design support, especially in the context of a virtual building design office.

\section{Keywords}

Integrated design, product modelling, interoperability, www-based data management

\section{OVERVIEW}

The EU ESPRIT project 6609 COMBI (Computer-integrated Object-oriented Model for the Building Industry) contributes essentially to a central prerequisite for an Internet-enabled co-operative design, namely the product model based 'integration through communication' (Scherer 1996).
\end{abstract}

The original version of this chapter was revised: The copyright line was incorrect. This has been corrected. The Erratum to this chapter is available at DOI: 10.1007/978-0-387-35390-6_58 
The fundamental objectives of COMBI were: (a) integration of traditional procedural and new intelligent design tools in a product modelling framework for co-operative design support, (b) application of knowledge-based and expert-system methodology to specific design tasks, e.g. in conceptual design, where static, procedural techniques fail, and (c) development of methods for effective data management and project co-ordination.

The heart of the COMBI environment is its modelling framework consisting of a layered set of product models represented by using object-oriented methodology. The distributed hierarchical architecture of the system and the applied representation methods allow a high flexibility for achieving inter-discipline interoperability and give powerful possibilities to integrate even non-object-oriented data structures of legacy off-the-shelve application tools. With a case implementation in conceptual structural design, soil and foundation engineering, structural analysis, element dimensioning and CADD, the COMBI prototype offers a methodology which is ready to serve as a platform for network-based design in a virtual design office, typically set up anew for each building project, with players from different enterprises, often SMEs with different information technology background and different used tools (Hannus et al. 1995).

AI methods are applied in COMBI to the cognitive problem of finding an appropriate structural load-bearing system starting from the conceptual architectural design. Decision support and classical expert-system technology are applied for the soil characterisation and the conceptual design of the foundation system. These component systems do all act as advanced 'suggest, select, modify' design systems.

With the integrated structural analysis and dimensioning system, two important integration aspects are addressed. First, it has been examined how traditional legacy tools with wide user acceptance can easily be upgraded for a high-level integration. Second, it has been demonstrated that locally integrated processes, such as numerical structural analysis and a layer-and-attribute based CAD system, can be effectively linked both separately and as one whole to an integrated product model environment.

The scope of COMBI was extended during the project phase with a product data management and control tool, and the COMBI methodology is already prepared for an extension to concurrent engineering. Key product model schemata for reinforced concrete structures have been developed and basic input to the architecture of the IFC project model (IFC 1997) has been provided. This paper presents the main concepts and techniques applied in PROMINENT, the manager's tool of the COMBI system.

\section{PROMINENT - THE PROJECT DATA MANAGEMENT TOOL}

\subsection{Product modelling}

The knowledge base of the integration framework is built up of a set of objectoriented product models. It is hierarchically structured in the following three levels of data abstraction and data reduction: 
- Application models, which are most detailed and contain specific application related objects.

- Partial aspect models, which are more general and depict the common properties of objects that have to be shared within each design domain.

- A neutral domain-independent kernel model, which - contrary to most other existing approaches - does not attempt to provide an integrating superset of the separate partial and application models, but serves rather for maintaining the consistence and the integrity of the product data instances in each specific design context, and for 'bridging' the different semantic representations of the modelling objects across disciplines and design domains.

This intentionally sought minimal common kernel is a unique feature of the COMBI framework which allows greatest autonomy in the development, implementation and future extension of the separate partial and application models, putting the emphasis of the integration strategy on the use of intelligent interoperability methods, rather than on a rigid semantic homogeneity of the representation.

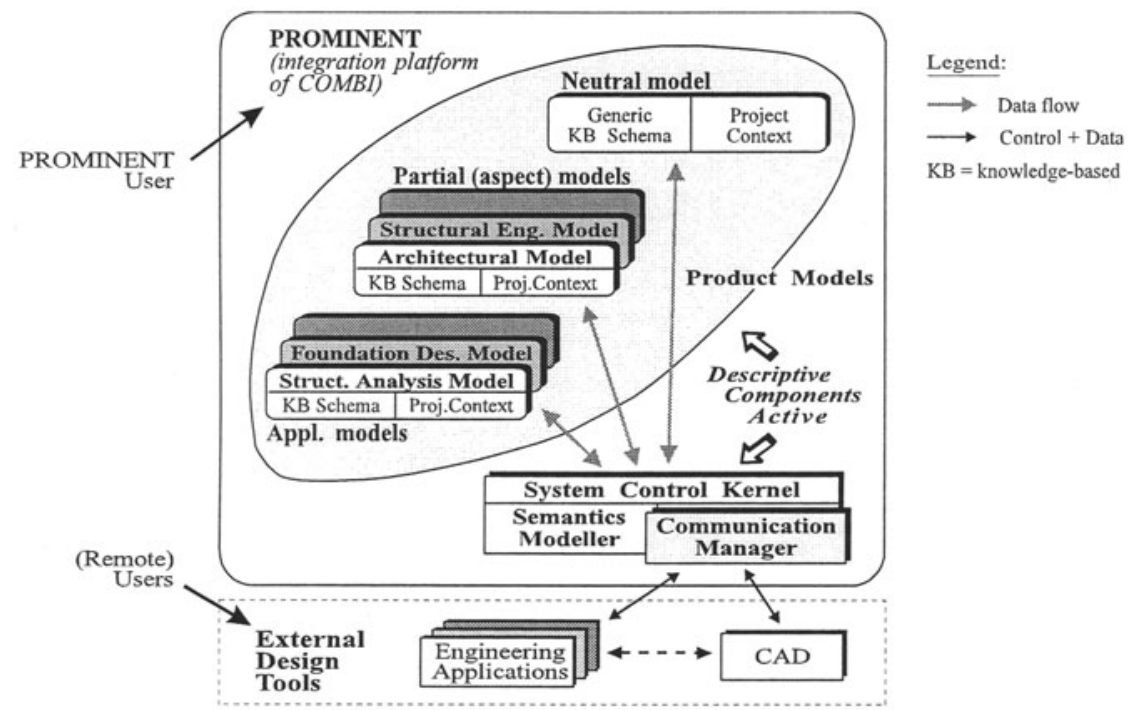

Figure 1: System architecture

Each model in the framework is by itself comprised of three interrelated components as shown in figure 1: the generic schema of the model, which provides the typological representation of engineering knowledge for the considered modelling perspective, an instantiated project context, which contains the actual instances of the modelling objects and is capable of representing the dynamic changes of the data during the design process, and active control methods, which support the needed data transformations, the communication with external applications etc. 


\subsection{Interoperability}

The separate models in the COMBI framework are specified formally in EXPRESS (ISO 10303-11). They use as far as possible the integrated resources defined in (ISO 10303-41, -42, -45). Interoperability is achieved with the help of a schema mapping language, which complements the EXPRESS specifications, and an active object matching mechanism installed in the communication management module of the system, which is responsible for the consistency of the product data.

Figure 2 shows schematically the full scope of the developed product models and the basic methods used for transforming the different semantic representations of the design information from one modelling space to another.

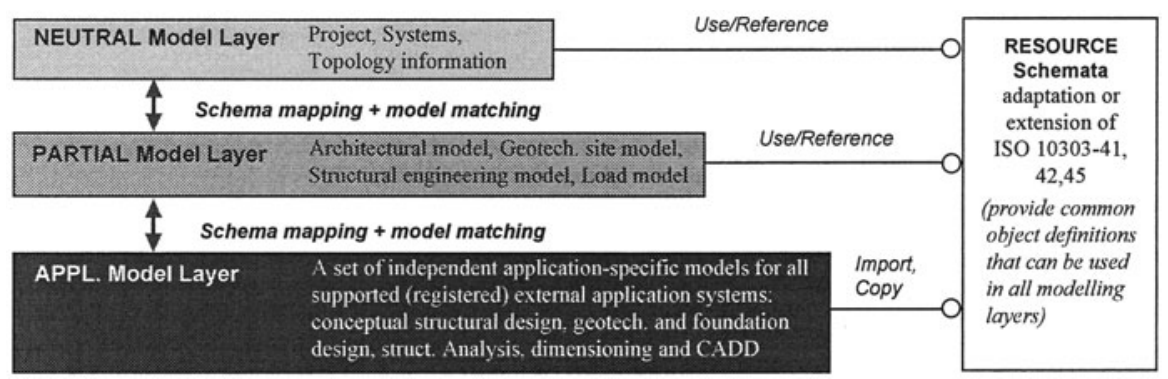

Figure 2: Product model schemata and their inter-relations in the framework

The actual product data exchange with each application is realised intentionally only with STEP physical files (ISO 10303-21), since this requires no (or only minimal) changes in the internal data structures of the applications. The whole data management process is identical for all integrated application tools and is controlled by the COMBI Communication Manager (CCM), as shown on figure 3 below. Its basic components are described in detail in the following sections.

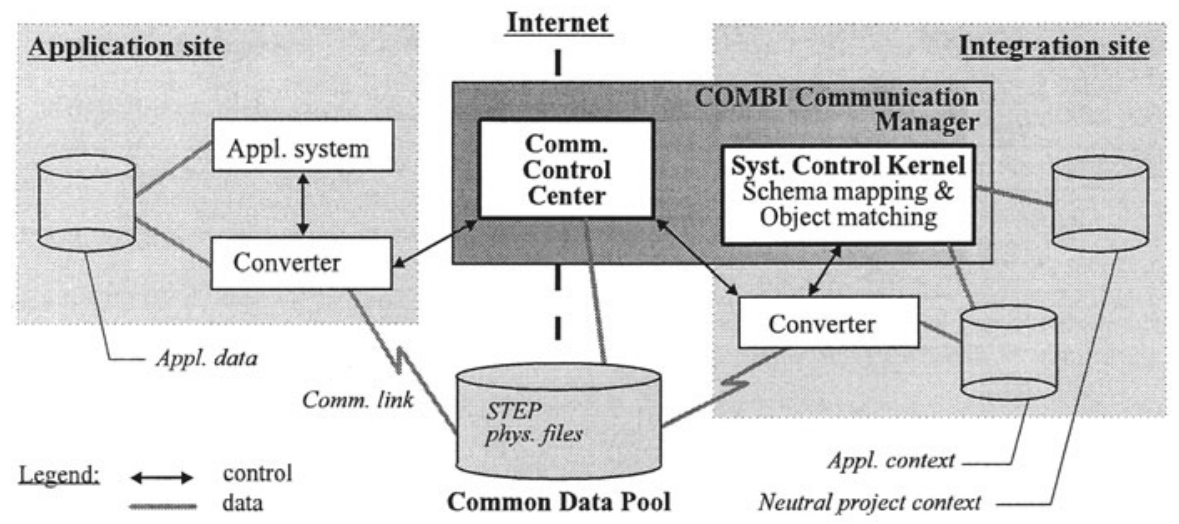

Figure 3: The data exchange process with an application on the basis of STEP files 


\subsection{Schema mapping}

The correspondences between the data belonging to different model spaces of the COMBI framework are defined formally with the help of a specially developed for that purpose schema mapping language (CSML). Despite the existing wide range of mapping languages today, for example EXPRESS-V (Hardwick 1994), EXPRESS-M (Bailey 1995), VML (Amor 1994) etc., we decided to create a new mapping language for COMBI for several reasons. First, all known languages have been developed in parallel or shortly before CSML and were still in an experimental phase. Second, they did not provide suitable enough programming language bindings. At last, it was found that all examined approaches target different interoperability areas, and whilst certainly useful in a number of concrete situations, none did match sufficiently the requirements of the COMBI integration approach, especially w.r.t. the effective use of a lean kernel model.

In our understanding, mapping is a one-directional data transformation process, serving to create new data to be used by an application. These can be: a new model, new object classes in an existing model, complete or partial transformations of object instances belonging to one model into object instances of another model.

CSML meets all these requirements. It uses a LISP-like notation style and contains constructs that provide means for partial and complete mappings of the entities defined in an EXPRESS schema, covering the cardinality cases 1:1, 1:0, $0: 1,1: \mathrm{C}, \mathrm{C}: 1,1: \mathrm{N}$ and (with some restrictions) M:N. A mapping specification in CSML consists of a header and a body that can contain the sections CLASSES, DEPENDENT-CLASSES, GLOBALS, FUNCTIONS. The most important and in fact the only obligatory of these sections is CLASSES. It is used to describe the inter-schema equivalences between the data in the source and the target model, whilst all other sections are used only in certain specific situations. CLASSES can itself encompass a number of mapping definitions, such as: CREATE-CLASS - which describes new classes (and respective instances) to be created in the target, MAP-CLASS - which expresses any kinds of $1: 1$ or $1: \mathrm{N}$ transformations, MAP-GROUP - which is a compound specification that may contain any number of the other 3 types and is used to describe M:N mappings etc. The main and most frequently used mapping construct is MAP-CLASS. Its syntax (in EBNF) is:

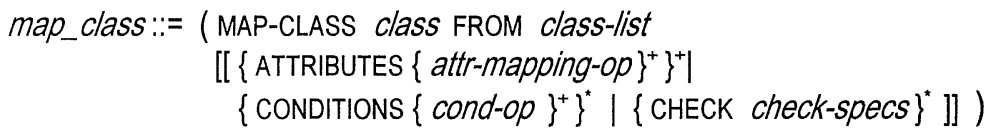

The individual clauses of MAP-CLASS have the following meaning. The FROM clause declares the objects in the source schema that have to be mapped. The CONDITIONS clause specifies when the MAP-CLASS definition can be applied. It may include one or more predicate functions which must all be true for the mapping to take place. The ATTRIBUTES clause specifies what are the equivalences between the attributes of the source object(s) and those of the target. These equivalence specifications can range from standard data type transformations to complex 
relations like chains of references, built-in, in-line and user-defined functions. At last, the CHECK clause specifies how to treat the new created target objects after the mapping operation. It contains also instructions used by the model matching tools.

CSML manifests an equivalent expressive power to the basic operands required in a relational database system (Ullman 1982) and thus can be easily used to perform all typical data transformations for database schemata, such as: projection, union, set difference, selection, Cartesian product, intersection etc.

\subsection{Model matching}

Though powerful enough to support both complete and partial model transformations, CSML alone is not sufficient to capture all dependencies and ensure the consistency of the product data in a project, because the static mapping specifications cannot take into account the dynamic changes to the modelling objects during the design process. Therefore, the implementation of CSML requires also an active data management component - model matching - that can track correctly the modifications of the data resulting from the mapping operations.

The necessity for matching object instances against the class specifications in the generic schemata of a product model arises when the mapping transformations are applied for updating already existing product information, e.g. in design interaction cycles such as between the architect, the structural engineer and the foundation designer etc.

In the integration environment of COMBI such problems are tackled by the specially developed object matching mechanism residing in the system control kernel. It is activated automatically whenever an application tries to update the corresponding information context, or when the results of a mapping operation have to be verified against the constraints defined in other relevant models.

The developed method follows the frame-based paradigm and is strongly related to the hierarchical architecture of the COMBI framework. Its key idea is to use the persistent neutral model of the framework as a reference structure for all other modelling representations by means of associating each object instance in a given context to instances of object primitives (or templates) specified in the neutral model. The class definitions of these object primitives cover only basic, domainindependent modelling aspects like the topological representation of the building elements, their implicitly maintained unique identification, generic object-to-object relationships like has-part, depends-on etc., whilst domain-specific aspects, including among others the explicit shape representation of all tangible building objects, are dealt with in the respective partial and application models.

Though labelled 'primitive', the generic classes defined in the neutral model are not simple. Besides attributes and methods determining their behaviour, they contain also specific rules which, when triggered, allow to recognise if an object instance can be associated to a particular class. To let these matching rules work on arbitrary objects whose structure is not known a priori, we use dynamic 'whenneeded' delegation links, as suggested by (Zucker and Demaid, 1992). In this way, 
a unified approach to all partial models is applied, and the matching rules are concentrated only in the neutral model, greatly simplifying the matching process.

\subsection{Communication Control}

In order to provide support for real co-operative design work, an integration environment has to include also a communication management layer which is capable of controlling the purpose of actor-to-actor communication, i.e. 'supervise who can do what and for what purpose at each stage of the design process'. Without such control, information exchange can easily become chaotic unless forcefully squeezed into a very restrictive schema.

In COMBI, this task is performed by the developed Communication Control Centre (CCC), which monitors all transactions to/from a common data pool via the Internet, using enabling World Wide Web CGI technology.

CCC is a relatively simple tool, which has been considerably enhanced in the COMBI follow-up project ToCEE (Scherer 1997). It is implemented entirely on the WWW and thus allows concurrent asynchronous access to the common data pool of a project by all project actors. CCC supports different process related operations such as: storing, retrieving, viewing STEP physical files (by using one of three alternative protocols - http, ftp or mail), posting messages to other participants in pre-specified formats, requesting summary reports of the state of the project. In order to provide control of the transactions between the distributed on the global network design actors, CCC uses an additional information layer containing: (1) a project description schema, which identifies the involved participants, their network addresses, roles, used tools etc., (2) a process description schema, which allows to determine the meaningful transactions and possible further design tasks by means of explicitly stored control items for each implemented model (extracted from its current context), and (3) a facts base, which stores all communication messages accompanying the product data exchange and facilitates reasoning about possible design actions.

\section{EXAMPLE FOR PRODUCT DATA EXCHANGE WITH MAPPING}

A simple practical example derived from the prototype implementation of COMBI can serve to illustrate the use of CSML (see figure 4). It depicts a portion of the mapping specifications needed to transform typical structural objects like nodes and nodal_results into objects for foundation design, which is necessary to allow the design cycle conceptual structural design $\Leftrightarrow$ foundation design to be performed properly, i.e. to transform reactions into loads and, inversely, the computed foundation stiffness into spring coefficients for frames and shear walls. This example gives a fairly good notion of the often very different representations of the building objects used by the different designers an integrated system has to deal with. Other more complex examples can be found in (Katranuschkov and Scherer, 1995). 


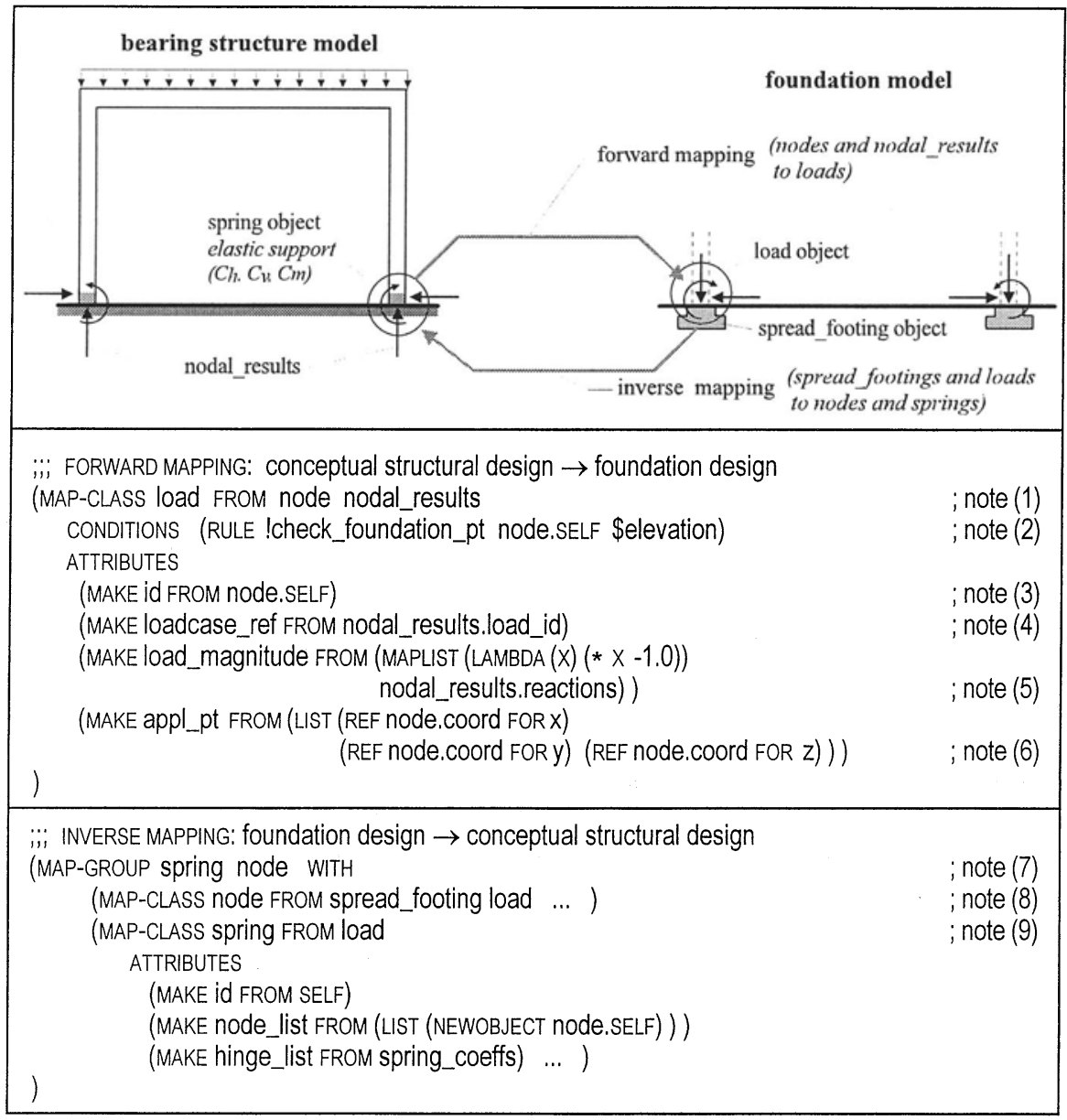

Notes:

(1) Starts the description of the inter-schema equivalences $b / n$ the classes load (in the source), and \{node, nodal_results\} in the target. (2) Applies a user-defined predicate function to the node entity and the global variable \$elevation to determine if the mapping of a particular node instance can proceed. In this case, requires that a structural node is a support and lies on a flat foundation with a pre-set elevation defined in the global coordinate system of the building. (3) Assigns the implicit entity identifier of the source (node) to the explicit attribute id of the target (load). (4) Performs simple attribute assignment. (5) Uses a built-in and an in-line function to transform a list of attribute values in the source instances to a respective list of values in the corresponding target instances.

(6) Constructs a list from three chained references: node $\rightarrow$ coord $\rightarrow x(y, z)$. (7) Starts the mapping specification for a group of classes and establishes a local scope for all their instances in the subsequent mapping declarations within the MAP-GROUP block. (8) Defines the mapping for the class node. (9) Defines the mapping for the class spring.

Figure 4: Mapping specification example 
The described integration methods are a promising beginning on the way towards STEP-based concurrent engineering systems. As proof of concept, they have been tested in a prototype environment with several knowledge-based system and conventional off-the-shelve applications as described in chapter 1 above. The obtained results have verified the potential power of the suggested approach. To give an impression of the system, on figure 5 below is presented a snapshot of the developed prototype integration platform PROMINENT, showing a run-time reflection of the example sketched on figure 4. It illustrates the WWW-based communication with the foundation designer, the GUI implemented as support environment for CSML (exposed on the main desktop of the integration platform), and the on-line interaction with a general-purpose CAD (presenting, in particular, the overlapping of the structural and the foundation model for visual evaluation of the solution - not possible in this form in any of the two involved design tools). The figure gives an impression of the practical benefits of the system for the endusers: co-ordinated and co-operative work using different tools, at remote places, integrated by a common modelling framework and enabling Internet technology.

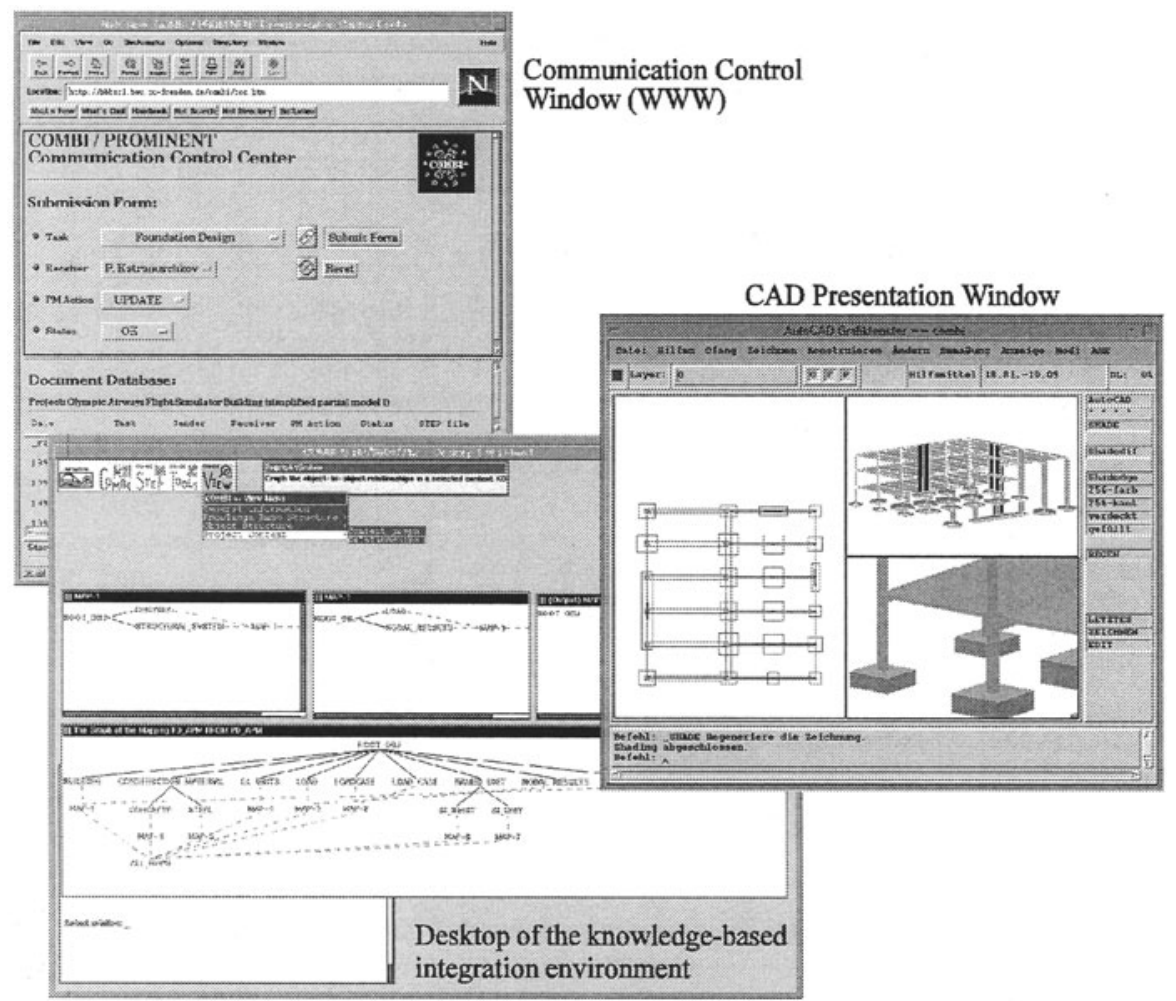

Figure 5: Snapshot of the COMBI integration platform 
The developed approach is a promising contribution on the way towards computer-aided collaborative product development. However, it has to be enhanced considerably in order to meet the requirements of a real concurrent engineering environment. Future development efforts have to be directed towards extending communication control to a full-scale information logistics service, enhancing the interoperability of the integrated system to include methods for conflict resolution, estimation of the impact of early design solutions on the whole life-cycle of a building, document management, auditing etc. These and other related research issues are being currently investigated in the follow-up EU ESPRIT project 20587 ToCEE, launched in January, 1996 (Scherer 1997). The funding of both the COMBI and the ToCEE projects by the European Commission has greatly facilitated this research work which is herewith deeply acknowledged.

\section{REFERENCES}

Amor R. (1994): A mapping language for views, Dept. Rep., Computer Science Dept., Univ. of Auckland, New Zealand.

Bailey I. (1995): EXPRESS-M reference manual, ISO TC184/SC4/WG5 N243.

Hannus M., Karstila K., Tarandi V. (1995): Requirements on standardised building product data models, in: Product and Process Modelling in the Building Industry (ed. R,J, Scherer), Proc. $1^{\text {st }}$ ECPPM'94, Dresden, Balkema Publ., Rotterdam.

Hardwick M. (1994): Towards integrated product databases using views, Rep. 94003, Design and Manufacturing Inst., Rensselaer Polytechnical Institute.

IFC Release 1.5 Final Version (1997): IFC object model for AEC projects, IAI Publ., Washington D.C.

ISO $10303-1,-11,-21,-41$ to -45 (1992-94): Product data representation and exchange, Parts 1, 11, 21, 22, 41-45, ISO TC184/SC4, Geneva.

Katranuschkov P. \& Scherer R.J. (1995): User manual of the product modelling integration environment for building design - PROMINENT v.1.1, Rep. 2/95, Inst. of Structural Mechanics and Applied Computer Science in Civil Eng., Dresden University of Technology.

Scherer R. J. (1996): COMBI final report, EU / CEC ESPRIT III Project No. 6609, Dresden University of Technology.

Scherer R. J. (1997): Overview of requirements and vision of ToCEE, Public Annual Report, EU / CEC ESPRIT IV Project No. 20587, Dresden University of Technology.

Ullman J. D. (1982): Principles of database systems, (second ed.), Computer Science Press.

Zucker J., Demaid A. (1992): Modelling heterogeneous engineering knowledge as transactions between delegating objects, in: Artificial Intelligence in Design '92, (ed. J. Gero), Kluwer Academic Publ., Rotterdam. 


\section{BIOGRAPHIES}

Prof. Dr.-Ing. Raimar J. Scherer is the head of the Chair of Applied Computer Science in Civil Engineering (CIB) at the Dresden University of Technology since April 1994. Before that he has worked at a building design office for 2 years, at the biggest German building CAD vendor for 2 years, and as full professor for $\mathrm{CAD} / \mathrm{CAM}$ in structural design at the University of Karlsruhe for 7 years. He was the technical manager of the ESPRIT Project COMBI and currently of the ESPRIT project TOCEE. He is president of the European Association on Product and Process Modelling in the Building Industry, member of the EG-SEA-AI, ISO TC184/SC4, the related German DIN-NAM 96.4.3 group and CALS-PDE, and is also vice chairman of the German DIN-CALS group and of the German DIN group of Technical Product Documentation.

Dipl.-Ing. Peter Katranuschkov works at the Dresden University of Technology since 1994. Currently he is senior research scientist at CIB. From 1992 to 1994 he was a member of Professor Scherer's research group at Karlsruhe. Before joining to the university he has been developing CAD software for the building industry for more than 10 years. He was a leading co-worker in the COMBI project and is currently involved in the TOCEE project. His experience comprises product and process modelling, expert systems, integration of engineering knowledge and the co-ordination of large research projects. 